\title{
Paxillin knockdown suppresses metastasis and epithelial-mesenchymal transition in colorectal cancer via the ERK signalling pathway
}

\author{
LONG WEN $^{1}$, XIAOQIAN ZHANG $^{1}$, JUNLING ZHANG ${ }^{1}$, SHANWEN CHEN $^{1}$, YONGCHEN MA ${ }^{2}$, \\ JIANWEN HU ${ }^{1}$, TAOHUA YUE ${ }^{1}$, JINGUI WANG ${ }^{1}$, JING ZHU ${ }^{1}$, TAO WU ${ }^{1}$ and XIN WANG ${ }^{1}$ \\ Departments of ${ }^{1}$ General Surgery and ${ }^{2}$ Endoscopic Center, Peking University First Hospital, Beijing 100034, P.R. China
}

Received November 13, 2019; Accepted May 28, 2020

DOI: $10.3892 / o r .2020 .7687$

\begin{abstract}
Paxillin (PXN) is a cytoplasmic protein that plays an important role in regulating focal adhesion, cytoskeletal rearrangements and cell motility. The present study aimed to investigate the role of PXN in the metastasis of human colorectal cancer (CRC) and its possible mechanisms. Immunohistochemical staining of tissues from 102 surgical CRC patients revealed that high PXN expression was positively correlated with tumour-node-metastasis (TNM) stage, lymph node metastasis, distant metastasis, and recurrence at distant sites after radical surgery. In 24 cases of stage IV CRC, PXN expression in liver metastasis was higher than that in the matched primary tumour. The knockdown of PXN inhibited the proliferation, migration and invasion potential of SW480 cells in vitro and in vivo. Transmission electron microscopy revealed the effect of PXN on ultrastructural characteristics, observed mainly in microvilli and desmosomes. The downregulation of PXN decreased the activation of extracellular regulated protein kinase (ERK) and suppressed the epithelial-mesenchymal transition (EMT) process. Following the downregulation of PXN, the addition of an ERK activator or inhibitor restored or further suppressed EMT, respectively, accompanied by corresponding changes in cell migration and invasion. Collectively, the present results confirmed the important role of PXN in CRC metastasis and revealed that PXN regulated EMT progression via the ERK signalling pathway. PXN may represent a future therapeutic strategy to prevent the EMT-associated progression and invasion of CRC.
\end{abstract}

Correspondence to: Professor Xin Wang, Department of General Surgery, Peking University First Hospital, Beijing 100034, P.R. China

E-mail: wangxin_guo@126.com

Key words: paxillin, colorectal cancer, metastasis, EMT, ERK

\section{Introduction}

Colorectal cancer (CRC) is the third most common cancer and fourth most common cause of cancer-related deaths globally (1). Metastatic dissemination, mainly to the liver and lungs, accounts for most CRC-related deaths (2). The pathological process and molecular mechanism of CRC metastasis are complex and not fully understood. The epithelial-mesenchymal transition (EMT), which involves the dissolution of cell-cell adhesions, loss of apical-basal lateral polarity and reorganization of the cytoskeleton, has been increasingly recognized to play pivotal and complex roles in promoting carcinoma metastasis $(3,4)$. Major developmental signalling pathways, including the TGF- $\beta$, Wnt, and growth factor receptor signalling cascades, have been demonstrated to be involved in some aspects of the EMT programme $(5,6)$. Similarly, the MAPK signalling pathway has been confirmed to have an impact on EMT in a variety of tumours, such as CRC, lung cancer and breast cancer (7-9).

Paxillin (PXN), a structural protein of $68 \mathrm{kDa}$, contains five leucine-aspartic acid (LD) motifs in its $\mathrm{N}$-terminal end that control most of its signalling activity and four LIM domains at its C-terminal end that mediate protein-protein interactions (10). As a focal adhesion protein, PXN was confirmed to play a prominent role in regulating cytoskeletal rearrangements, tissue remodelling and cell motility (11). PXN expression has been revealed to be negatively correlated with CRC patient prognosis $(12,13)$. The phosphorylation of PXN, which is regulated by multiple mRNAs, can promote CRC cell invasion (14). However, the influence of PXN on EMT and the underlying mechanism by which $\mathrm{PXN}$ promotes metastasis in CRC remain unknown.

In present study, data was collected from 102 postoperative patients. Immunohistochemistry was performed to evaluate the relationships between PXN expression and N stage, $\mathrm{M}$ stage and distant site recurrence. Furthermore, differences in PXN expression between the primary tumour and matched liver metastasis specimens from 24 patients who underwent concurrent resection were analysed. SW480 cells were selected to clarify the role of PXN in cell proliferation, migration and invasion. The relationship between PXN and EMT was explored, and the results revealed that the extracellular 
signal regulated kinase (ERK) signalling pathway may act as an intermediate between PXN and EMT.

\section{Materials and methods}

Tumour samples. The retrospective study was approved by the Peking University First Hospital Biomedical Research Ethics Committee (No. 2018-15). CRC samples were collected from the Department of Surgery at Peking University First Hospital between January 2010 and December 2012. All patients related to this study signed an informed consent agreement before surgery. The present study enrolled the patients (including 56 males and 46 females, with a median age of 68 years) with primary colorectal cancer diagnosed by pathology, and excluded those with other tumours or that had received radiotherapy and chemotherapy before surgery. The clinicopathologic stage was determined according to TNM classification [American Joint Commission on Cancer (AJCC) 8th edition]. Follow-up data were collected by telephone and outpatient interview.

Cell lines and reagents. The SW480, SW620, Caco-2, DLD-1, HCT116, and LoVo human colon cancer cell lines were obtained from the General Surgery Laboratory of Peking University First Hospital (Beijing, China). These cells were routinely cultured in Dulbecco's modified Eagle's medium (DMEM) with $10 \%$ foetal bovine serum (FBS; both from Gibco; Thermo Fisher Scientific, Inc.) at $37^{\circ} \mathrm{C}$ and supplemented with $5 \% \mathrm{CO}_{2}$. hEGF (cat. no. E9644), which was used as an ERK1/2 activator in our research (15), was purchased from Sigma-Aldrich; Merck KGaA. SCH-772984, a selective inhibitor of Erk1/2 (hereinafter called EI), dissolved in dimethylsulfoxide (DMSO) was purchased from Selleckchem. Both hEGF and EI were maintained at $-20^{\circ} \mathrm{C}$.

Immunohistochemical staining. Paraffin-embedded tumour tissues were cut into 5- $\mu$ m-thick sections. Anti-PXN antibody (1:100 dilution; product code ab2264; Abcam) and horseradish peroxidase (HRP)-conjugated goat anti-rabbit IgG (cat. no. PV6001; ZSGB-BIO) were used as primary and secondary antibodies, respectively. DAB staining (5 min at room temperature; cat. no. ZLI-9018; ZSGB-BIO) was used to visualize PXN expression, which was evaluated independently by a pathologist and researcher and scored based on the proportion of deposited cells with a brown granular appearance as follows: $<10 \%$, score of $0 ; 11$ to $25 \%$, score of $1 ; 26$ to $50 \%$, score of $2 ; 51$ to $75 \%$, score of 3 ; and 76 to $100 \%$, score of 4 . The staining intensity was assessed and scored as follows: Negative staining, score of 0 ; mild positive staining, score of 1 ; moderate positive staining, score of 2 ; and strong positive staining, score of 3 . The total score was equal to the score indicating the proportion of positive cells multiplied by the score indicating the staining intensity. Tumour tissues with a total score of 4 or more were categorized as expressing high levels of PXN, while those with a score less than 4 were categorized as expressing low levels of PXN.

Cell transfection. We searched in BLAST (Basic Local Alignment Search Tool; https://blast.ncbi.nlm.nih.gov/Blast.cgi) and designed two short hairpin RNAs (shRNA), which specifically knock down the mRNA of PXN. Plasmids expressing shRNAs specifically targeting PXN (shPXN) and a negative control shRNA (shNC) were constructed by Shanghai GenePharma Co., Ltd. The shPXN sequences were 5'-GGGCAGCAACCT TTCTGAACT-3' (shPXN1) and 5'-GGAGAGTCTCTTGG ATGAACT-3' (shPXN2). Lipofectamine 3000 (Invitrogen; Thermo Fisher Scientific, Inc.) was used for transfection. Cell clones stably expressing shRNA were selected with G418 (Geneticin; Gibco Thermo Fisher Scientific, Inc.).

Cell proliferation. Cancer cell proliferation was evaluated using the Cell Counting Kit-8 assay (cat. no. B34304; Bimake). SW480 cells were seeded in 96 -well plates $\left(5 \times 10^{3}\right.$ cells $/$ well $)$ and incubated for $1,2,3$ or 4 days at $37^{\circ} \mathrm{C}$. Then, CCK-8 reagent $(10 \mu \mathrm{l})$ was added to each well. Each plate was incubated for another $1.5 \mathrm{~h}$ at $37^{\circ} \mathrm{C}$, and the absorbance at $450 \mathrm{~nm}$ was measured. Each experiment was repeated at least three times.

Colony formation assay. SW480 cells were counted and seeded in 6-well plates at 200 cells/well. The culture medium was replaced every 3 days over a 2 -week incubation. At the time of harvesting, the medium was removed, and colonies were stained with $0.05 \%$ crystal violet for $1 \mathrm{~h}$ at room temperature. Samples were allowed to dry at room temperature, and each plate was photographed with a camera (A7M3; Sony). Each colony was counted only if it contained more than 50 cells by using ImageJ software (v1.4.3.67; National Institutes of Health). Each experiment was performed in triplicate.

Wound healing assay. Equivalent numbers of SW480 cells $\left(n=5 \times 10^{5}\right)$ were seeded in 6 -well plates, and wounds were generated in the cells covering the plate. Each well was cultured with serum-free DMEM and the appropriate drugs (hEGF and EI) for $48 \mathrm{~h}$ and imaged over time. The scratched area was captured by a light microscope (magnification, $\mathrm{x} 40$ ) and measured using ImageJ software (v1.4.3.67; National Institutes of Health) by finding the edges of the wound. Each sample was assessed in three fields as three replicates.

Transwell assays. For the migration assay, a Transwell chamber with $8-\mu \mathrm{m}$ pores (BD Biosciences) was used to establish a bilayer culture model. The upper chamber was seeded with $1 \times 10^{5} \mathrm{SW} 480$ cells in serum-free DMEM, while $600 \mu \mathrm{l}$ of medium containing $20 \%$ FBS was added to the lower chamber. After $24 \mathrm{~h}$ of incubation at $37^{\circ} \mathrm{C}$, the cells that had migrated through the filter were fixed with $100 \%$ methanol for $20 \mathrm{~min}$ and stained with $0.05 \%$ crystal violet for $10 \mathrm{~min}$, at room temperature. Non-migrated cells on the top surface of the filter were removed, and migrated cells were observed with a light microscope (magnification, x100) and counted with ImageJ software (v1.4.3.67; National Institutes of Health). The Transwell invasion assay was performed in a similar manner, except that the top chamber was pre-coated with $50 \mu \mathrm{l}$ of Matrigel (1:8 dilution with DMEM; Corning, Inc.). Each experiment was repeated at least three times.

Western blot analysis. Total cellular protein and phosphorylated protein samples were prepared from cell lysates (SW480, SW620, Caco-2, DLD-1, HCT116 and LoVo cells) in lysis buffer (cat. no. KGP2100; Nanjing KeyGen Biotech, Co., Ltd.). 
The protein determination of samples was performed by BCA method. After the protein concentration of each sample was adjusted, 10\% sodium dodecyl sulfate (SDS)-polyacrylamide gel electrophoresis was performed to separate the proteins (20 $\mu \mathrm{g}$ per lane). Subsequently, the protein bands were transferred to polyvinylidene difluoride (PVDF) membranes, and blocked using 5\% BSA (Sigma-Aldrich) at room temperature for $1 \mathrm{~h}$. The membranes were incubated with specific primary antibodies against PXN (product no. 2542), phosphorylated (p)-ERK (product no. 4370), ERK (product no. 4695), vimentin (product no. 5741), Snail (product no. 3879), $\mathrm{N}$-cadherin (product no. 13116), E-cadherin (product no. 3195) and GAPDH (product no. 5174) at $4^{\circ} \mathrm{C}$ overnight. All of the aforementioned primary antibodies were purchased from CST and used at a 1:1,000 dilution. GAPDH was used as an internal control. Horseradish peroxidase (HRP)-conjugated goat anti-rabbit IgG (cat. no. ZB-2301; ZSGB-BIO; OriGene Technologies, Inc.) diluted with BSA and diluted 1:10,000 was used as a secondary antibody. The expression levels of the target proteins were assessed via an electrochemiluminescence (ECL) detection system (Merck KGaA) on a Syngene Gene Genius gel imaging system (Syngene Europe).

In vivo assay. The animal experiments were approved by the Laboratory Animal Ethics Committee of Peking University First Hospital (No. 2015-55). Male BALB/c nude mice $(n=24$; weight 18-20 g) at 4-5 weeks of age were purchased from Beijing Vital River Co., Ltd. and maintained at SPF (specific pathogen-free) laboratory barrier facility of the Experimental Animal Center of Peking University First Hospital, with controlled temperature and humidity conditions $\left(22 \pm 1^{\circ} \mathrm{C}\right.$; 40-60\%) with food and water ad libitum. SW480 cells stably expressing shNC and shPXN $\left(1 \times 10^{7}\right.$ cells per mouse, $n=6 /$ group) were injected subcutaneously into the left flanks of the nude mice to establish a tumour xenograft model. The tumour volume was measured every 3 days and calculated as width $^{2} \mathrm{x}$ length $/ 2$. After 6 weeks, the mice were sacrificed by cervical dislocation, and the tumours were excised for further study. Another group of nude mice was randomly divided into two groups ( $\mathrm{n}=6 /$ group), and two groups of SW480 cells transfected with shRNA ( $1 \times 10^{5}$ cells per mouse) were individually injected via the tail vein to establish lung experimental metastasis models. After 5 weeks, the mice were sacrificed by cervical dislocation, and their major organs (lung and liver) were collected and embedded in paraffin. Organ samples were sliced into five continuous sheets at the maximum cross-sectional area, embedded in paraffin and stained with haematoxylin and eosin $(\mathrm{H} \& \mathrm{E}$, at room temperature for 5 and $1 \mathrm{~min}$, respectively). Metastases in all sections observed by a light microscope (magnification, x200) were counted and averaged.

Transmission electron microscopy. The subcutaneous tumours from the nude mice were cut into $1 \times 1 \mathrm{~mm}$ pieces within one minute after separation and fixed in $3 \%$ glutaraldehyde at $4{ }^{\circ} \mathrm{C}$ for $24 \mathrm{~h}$. Subsequent specimen preparation was completed by the Department of Electron Microscopy of Peking University First Hospital. Transmission electron microscopy images were captured using a JEM-1230 microscope (JEOL, Ltd.). Image acquisition and analysis were completed using
DigitalMicrograph software (v1.8.3; Gatan, Inc.) under the guidance of a pathologist specializing in electron microscopy.

Immunofluorescence (IF) staining. An equivalent amount of SW480 cells $\left(n=5 \times 10^{5}\right)$ was seeded on sterilized glass coverslips. Briefly, after fixation with methanol for $20 \mathrm{~min}$ and blocking with goat serum (1:10 dilution; cat. no. ZLI-9022; ZSGB-BIO) for $1 \mathrm{~h}$ at room temperature, the coverslips were incubated with primary antibodies against vimentin, N-cadherin and E-cadherin (1:100 dilution; product nos. 5741, 13116 and 14472, respectively; Cell Signalling Technology) overnight at $4^{\circ} \mathrm{C}$, followed by Alexa 488-conjugated goat anti-rabbit antibody and Alexa 555-conjugated goat anti-mouse antibody (1:100 dilution; product codes A11008 and A21422, respectively; Invitrogen; Thermo Fisher Scientific, Inc.) for $1 \mathrm{~h}$ at room temperature. The nuclei were stained with 4',6-diamidino-2-phenylindole (DAPI; ZLI-9557; ZSGB-BIO) for $30 \mathrm{~min}$ at room temperature. The fluorescent expression of the target markers and the nuclei were evaluated and imaged using a Leica confocal laser microscope (magnification, x400).

Statistical analysis. Statistical data analyses were performed using SPSS 24.0 statistical software (IBM Corp.). The values in this study are reported as the mean \pm standard deviation (SD) and are representative of an average of at least three independent experiments. Student's t-test and repeated measures analysis of variance (ANOVA) followed by LSD or Tukey's post hoc tests were used for statistical analyses. The $\chi^{2}$ test was performed to evaluate the association between the expression level of PXN and clinicopathological parameters. $\mathrm{P}<0.05$ was used to indicate a statistically significant difference.

\section{Results}

PXN expression is associated with clinical pathological parameters in CRC metastasis. There were 102 patients enrolled in the present study, including 56 males and 46 females, with a median age of 68 years. Of the patients, 16 , 26, 36 and 24 patients had pathological stage I, stage II, stage III and stage IV CRC, respectively. As revealed in Table I, PXN expression was significantly associated with $\mathrm{N}$ stage $(\mathrm{P}=0.014)$ and $\mathrm{M}$ stage $(\mathrm{P}=0.007)$, but there was no significant difference depending on age or sex $(\mathrm{P}>0.05)$. The proportion of patients with stage III and stage IV CRC exhibiting high PXN expression was higher than that of patients with stage I and stage II CRC ( 80 vs. $54.8 \%$, respectively, $\mathrm{P}=0.006$ ). Three-year follow-up data revealed that in all patients who underwent R0 resection, the proportion of metastatic patients with high PXN expression was higher than that of non-metastatic patients (88.5 vs. $60.6 \%$, respectively, $\mathrm{P}=0.010$ ).

Stage IV CRC patients exhibit higher PXN expression in liver metastases than in primary tumours. Twenty-four paired specimens from patients with stage IV CRC who underwent concurrent excision of the primary lesion and liver metastasis were included. The PXN protein was expressed mainly in the cytoplasm (Fig. 1A). The total score reflecting PXN expression was significantly higher in liver metastases than in paired primary lesions $(9.08 \pm 2.38$ vs. $6.46 \pm 2.57$, respectively, 
Table I. The expression of PXN is associated with clinicopathological parameters in CRC metastasis.

\begin{tabular}{lcccr}
\hline Clinicopathological parameters & Total number & Low PXN expression n (\%) & High PXN expression n (\%) & P-value \\
\hline Age (years, median, quartiles) & 102 & $70(66,74)$ & $67(53,74)$ & 0.111 \\
Sex & & & & 0.382 \\
Male & 56 & $15(26.8)$ & $41(73.2)$ & \\
Female & 46 & $16(34.8)$ & $30(65.2)$ & 0.014 \\
N stage & 44 & $19(43.2)$ & $25(56.8)$ & 0.007 \\
N0 & 58 & $12(20.7)$ & $46(79.3)$ & \\
N+ & 78 & $29(37.2)$ & $49(62.8)$ & 0.006 \\
M stage & 24 & $2(8.3)$ & $22(91.7)$ & \\
M0 & & & $23(54.8)$ & \\
M1 & 42 & $19(45.2)$ & $48(80.0)$ & \\
TNM stage & 60 & $12(20.0)$ & $40(60.6)$ & \\
I/II & & $26(39.4)$ & $23(88.5)$ & \\
III/IV & 66 & $3(11.5)$ & & \\
Recurrence of distant site & 26 & & & \\
No & & & & \\
Yes & & & & \\
\hline
\end{tabular}

${ }^{a}$ Only included patients who underwent R0 resection; follow-up time was three years. PXN, paxillin; CRC, colorectal cancer.

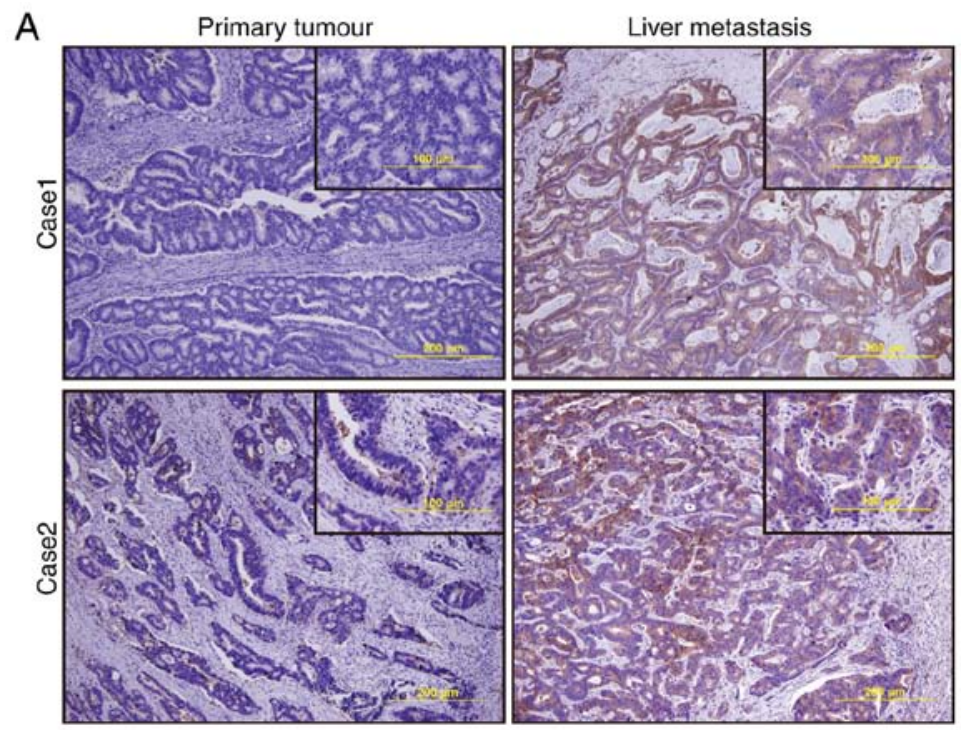

B

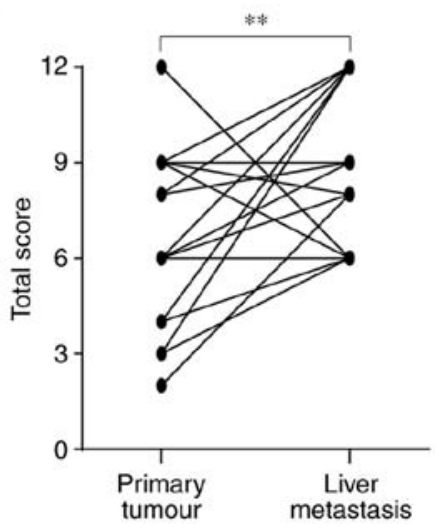

Figure 1. PXN expression in liver metastases is higher than that in primary tumours. (A) PXN expression in primary colorectal adenocarcinoma and paired liver metastasis was assessed by immunohistochemistry (original magnification, $\mathrm{x} 100$; detailed magnification, $\mathrm{x} 200$ ). (B) Comparison of PXN expression in primary and metastatic lesions from 24 cases. PXN expression in liver metastasis was equal to or higher than that in the primary tumour in 22 cases, as revealed by the connection diagram. P-values were obtained by paired-sample Student's t-test. ${ }^{* *} \mathrm{P}<0.01$. PXN, paxillin.

$\mathrm{P}=0.002$, Fig. 1B). The number of cancer-associated fibroblasts (CAFs) in liver metastasis was counted, however, there was no significant difference between the number of CAFs and PXN expression (Fig. S1).

Knockdown of PXN suppresses the proliferation, migration and invasion potential of SW480 cells. Among Caco-2, LoVo, SW480, HCT116, DLD-1 and SW620 cells, PXN was expressed at the highest level in SW480 cells, which were used for subsequent experiments (Fig. 2A). Compared with SW480 cells transfected with shNC, which exhibited no difference in PXN expression compared with control SW480 cells, SW480 cells transfected with shPXN exhibited significantly reduced PXN expression (Fig. 2B). shPXN1 was selected for subsequent experiments in the present study since it more effectively suppressed PXN expression.

With PXN knockdown, cells exhibited a more rounded appearance (Fig. 2C), and the proportion of migrated cells 
A
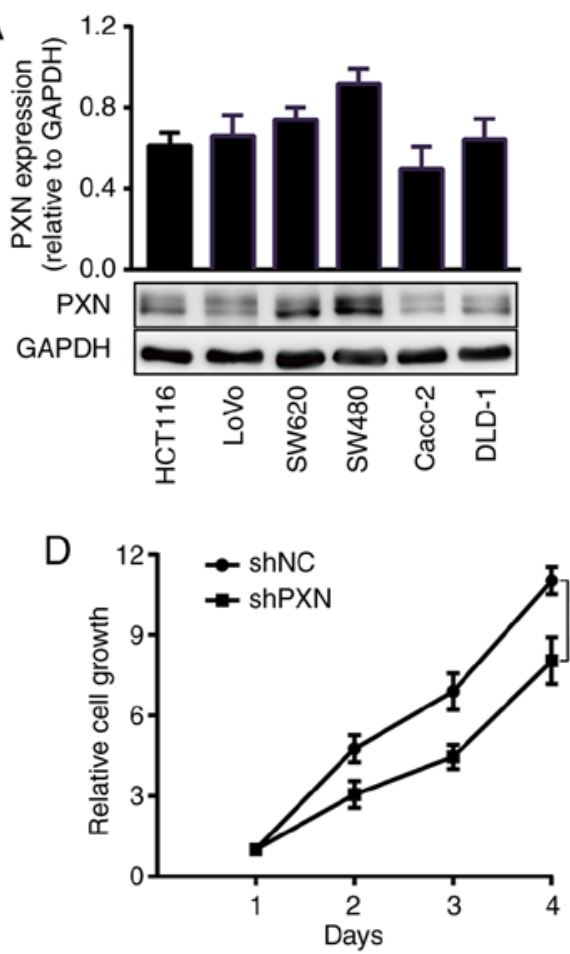

F
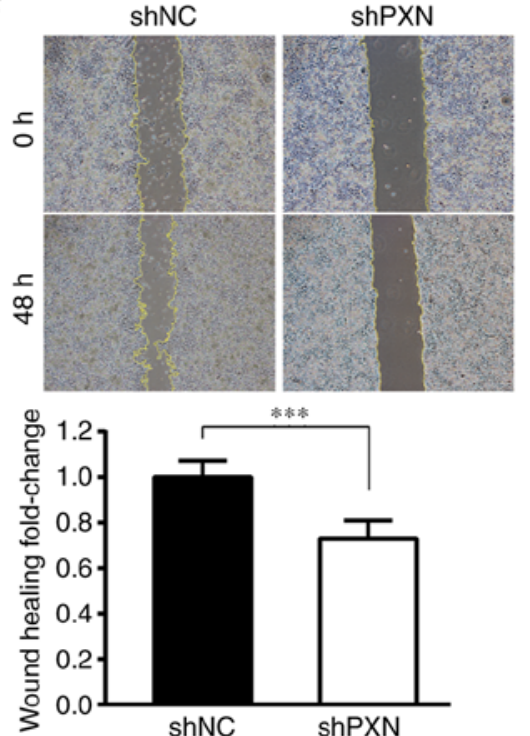

$\mathrm{B}$
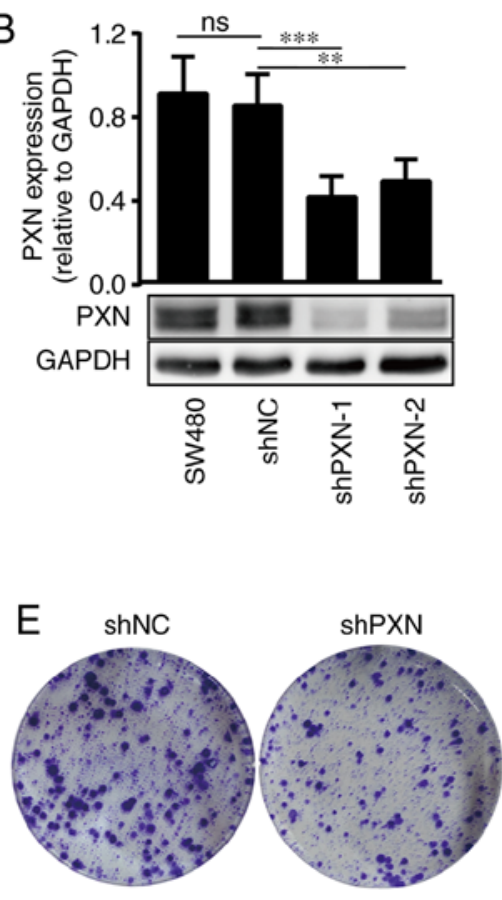

G
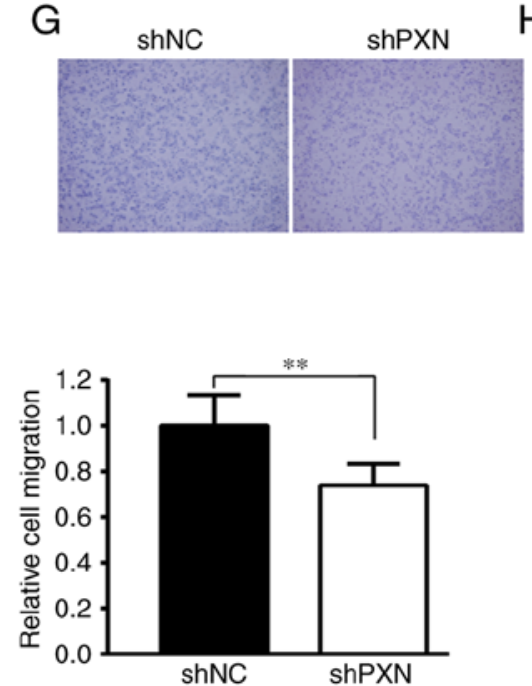
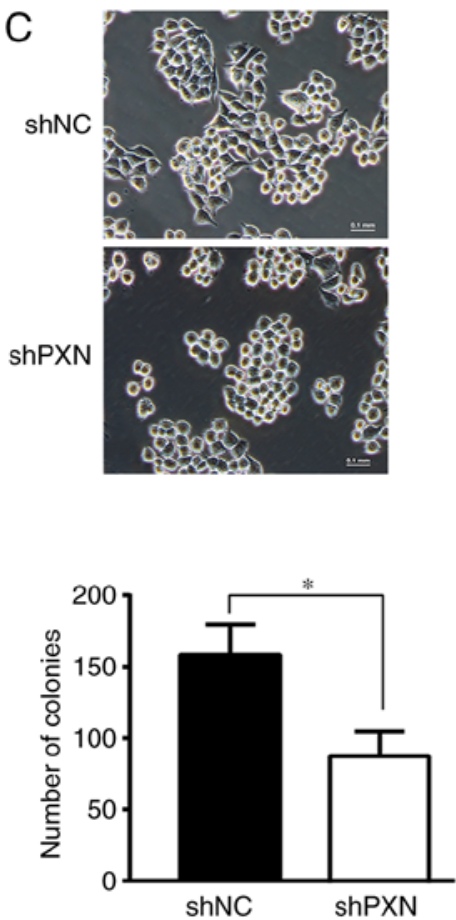

Figure 2. Knockdown of PXN suppresses the proliferation, migration and invasion potential of SW480 cells. (A) Diverse expression levels of PXN in colorectal adenocarcinoma cell lines analysed by western blotting. The histogram represents the relative protein expression. (B) Downregulation of PXN in SW480 cells. Cells were transfected with a non-specific control (shNC) or one of two shRNAs targeting PXN (shPXN). The knockdown efficiency was verified by western blotting. (C) Changes in cell morphology (magnification, x200). (D) The knockdown of PXN inhibited the proliferation of SW480 cells. CCK-8 assays were used to assess the proliferation at different time-points. The data are presented as the mean \pm SD of at least three independent experiments. P-values were obtained by repeated measures ANOVA (LSD post hoc test). (E) The knockdown of PXN inhibited colony formation in SW480 cells. The number of colonies per plate was counted using ImageJ software. ( F and G) The knockdown of PXN inhibited SW480 cell migration. The migration ability of cells was measured using (F) wound healing and (G) Transwell migration assays. Changes in the wound width at $48 \mathrm{~h}$ after the scratch was calculated using Image J software. The histogram represents the change in wound width and the number of migrated cells relative to the shNC group. (H) The knockdown of PXN inhibited SW480 cell invasion. Invasion ability was assessed using the Transwell invasion assay. Cells that penetrated the Matrigel were counted. The data are presented as the mean \pm SD of at least three independent experiments. P-values were obtained by Student's $t$-test. ${ }^{*} \mathrm{P}<0.05,{ }^{* *} \mathrm{P}<0.01,{ }^{* * *} \mathrm{P}<0.001$. PXN , paxillin; CCK-8, Cell Counting Kit-8; ns, no significance.

decreased by approximately $30 \%$ (Fig. $2 \mathrm{~F}$ and G). The invasion assay revealed that the number of cells that penetrated the Matrigel was decreased by more than half (Fig. 2H). Decreased PXN expression also inhibited cell proliferation (Fig. 2D) and colony formation $(\mathrm{n}=84.67 \pm 13.53$ vs. $151.67 \pm 20.59$, respectively, $\mathrm{P}=0.011$, Fig. 2E).
Downregulation of PXN inhibits tumourigenesis in vivo. Next, the effects of PXN on tumour proliferation and metastasis in vivo were explored. The tumour mass was palpable on approximately the tenth day. As revealed in Fig. 3A, the mice in the shNC group exhibited faster tumour growth. In contrast, PXN downregulation resulted in a smaller tumour volume 

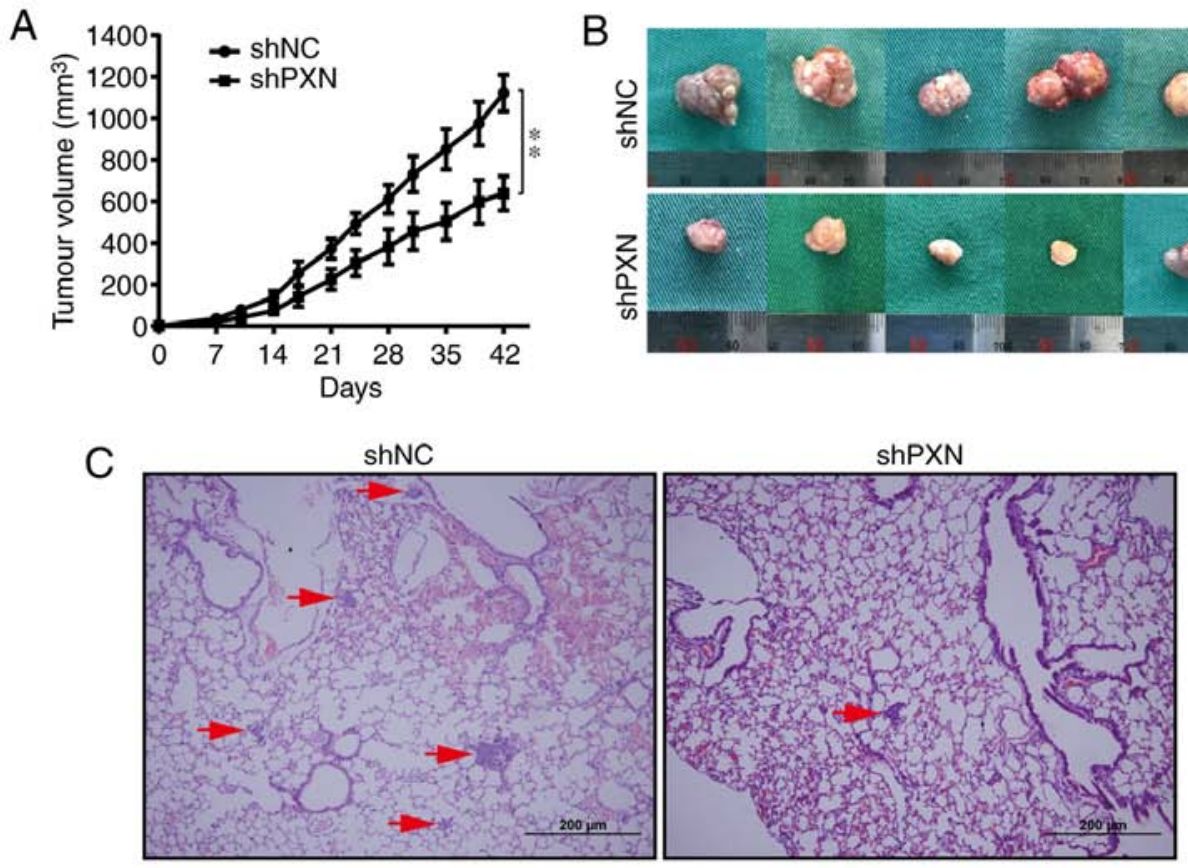

B

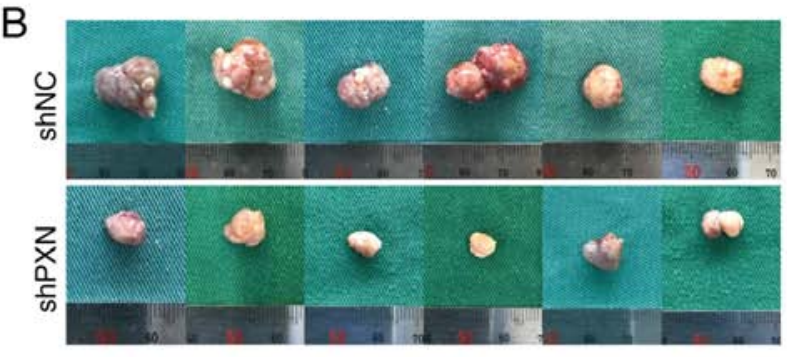

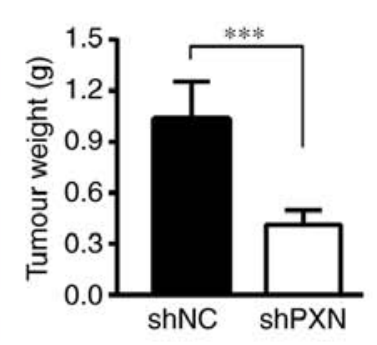

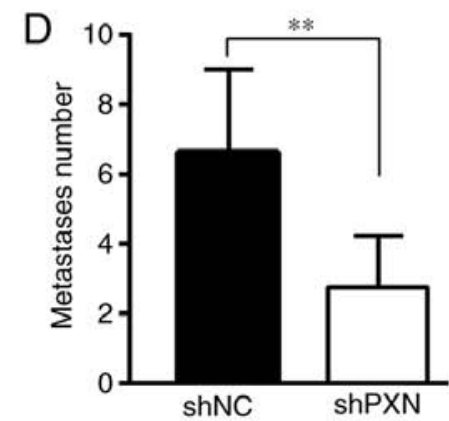

Figure 3. Downregulation of PXN inhibits tumourigenesis in vivo. (A) Tumour growth curves. SW480 cells stably expressing shNC and shPXN were injected subcutaneously into nude mice to form subcutaneous tumour models $(n=6)$. The rate of subcutaneous tumour growth in the shPXN group was slower than that in the shNC group. P-values were obtained by repeated measures ANOVA (LSD post hoc). (B) Tumour weight. Tumours were dissected and measured at the end point. (C) Lung experimental metastases observed by microscopy (original magnification, x100). The red arrow indicates experimental metastases in the lung acinus. (D) The number of experimental metastases in the acini and bronchial wall was counted. P-values were obtained by Student's t-test. ${ }^{* *} \mathrm{P}<0.01$, ${ }^{* * * *} \mathrm{P}<0.001$. PXN, paxillin.

$\left(637.28 \pm 82.87\right.$ vs. $\left.1120.72 \pm 88.39 \mathrm{~mm}^{3}\right)$ and decreased tumour weight $(0.41 \pm 0.09$ vs. $1.04 \pm 0.22 \mathrm{mg}$, Fig. $3 \mathrm{~B})$ over the same growth period, which was in accordance with the results of the in vitro experiments.

Tumour cells were injected via the tail vein to observe the effect of PXN on experimental metastasis in vivo. No metastases were found in the liver. Metastatic nodules on the lung specimens were not macroscopic but could be clearly observed and quantified under a microscope. The lung metastases of the shNC group were larger and denser, and some masses appeared next to the bronchi, while the tumour sites in the shPXN group were mainly at the acini (Fig. 3C). There were significantly fewer experimental metastases in the shPXN group than in the shNC group ( $n=2.75 \pm 1.49$ vs. $6.62 \pm 2.38$, respectively, Fig. 3D).

Knockdown of PXN reverses malignant ultrastructural characteristics. In the shNC group, numerous microvilli, a bulging arrangement of individual cells, formed on the cell surface, and glandular lumens were observed between cells in partial microscopic fields (Fig. 4A-a). These microvilli were rarely found on the surface of shPXN-transfected cells, leading to a smooth cytomembrane (Fig. 4A-b). Cell-cell contacts and junction structures are presented at a higher magnification. The connections between shNC-transfected cells, which were maintained mainly by desmosomes and tight connections, were no longer tight (Fig. 4A-c). In contrast, the shPXN-transfected cells were attached and closer, and their junction structures were clearer (Fig. 4A-d). As illustrated in Fig. 4B, PXN knockdown induced an increase in the number of desmosomes $(n=3.20 \pm 0.39$ vs. $1.90 \pm 0.23$, respectively, $\mathrm{P}=0.01$ ).
PXN regulates the expression of EMT markers. Taking into consideration the change in cell migration ability, morphology and ultrastructural characteristics induced by the downregulation of PXN, it was hypothesized that PXN plays an important role in EMT progression and evaluated the expression of EMT markers using western blotting (WB) and IF. As revealed in Fig. 4C, PXN knockdown decreased the expression of $\mathrm{N}$-cadherin and vimentin, two classic mesenchymal markers, and increased the expression of E-cadherin. In addition, the expression of Snail, a transcription factor that suppresses E-cadherin, was decreased following PXN knockdown. The IF images of E-cadherin, N-cadherin and vimentin (Fig. 4D) exhibited a similar expression change as that observed in the WB experiments. Both types of cells exhibited positive membranous and cytoplasmic immunoreactivity for E-cadherin. Notably, knockdown of PXN resulted in increased and more continuous expression of E-cadherin on the cytomembrane, followed by a tighter cell connection, which was consistent with the observation in TEM images.

PXN regulates the ERK signalling pathway. To further explore how PXN regulates EMT, its effects were verified on two classic pathways, the ERK and $\beta$-catenin signalling pathways. As revealed in Fig. 5, PXN downregulation decreased the expression of p-ERK, whereas total ERK1/2 levels remained unchanged, indicating the loss of ERK activation. In contrast, the knockdown of PXN did not change the expression of $\beta$-catenin. These results demonstrated that PXN silencing could decrease the activity of the downstream ERK signalling pathway. 
A a

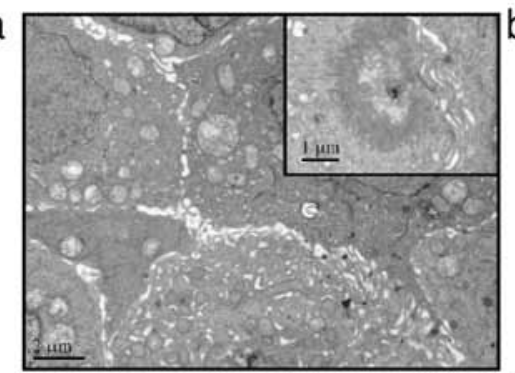

C
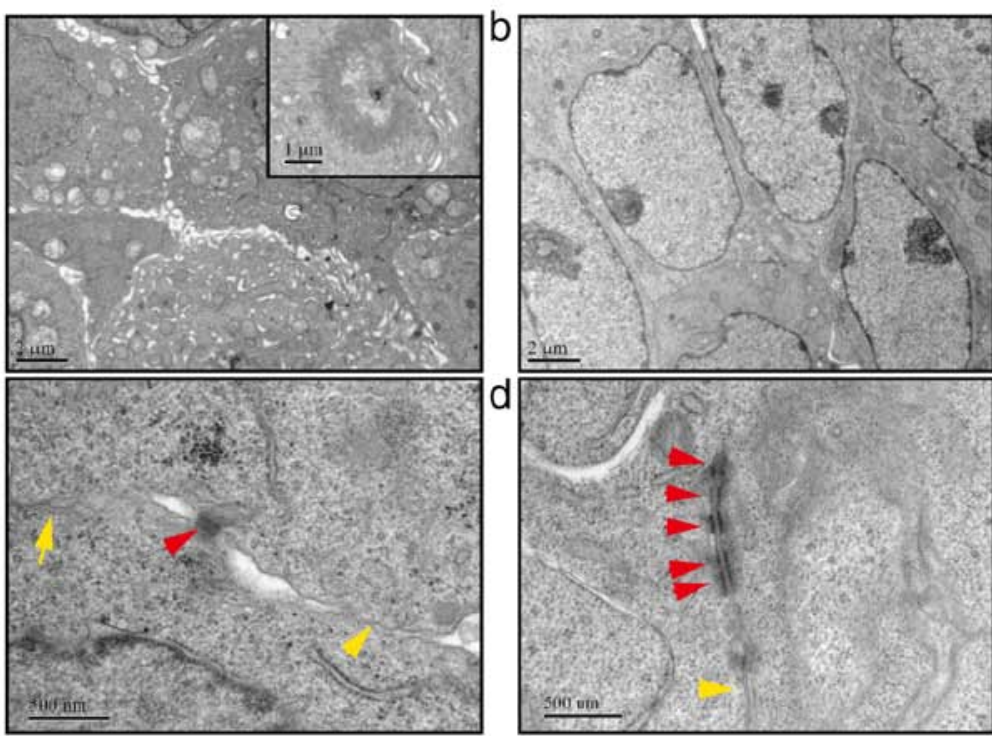

$\mathrm{D}$ a

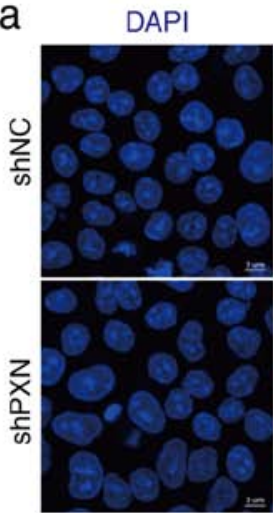

E-cadherin
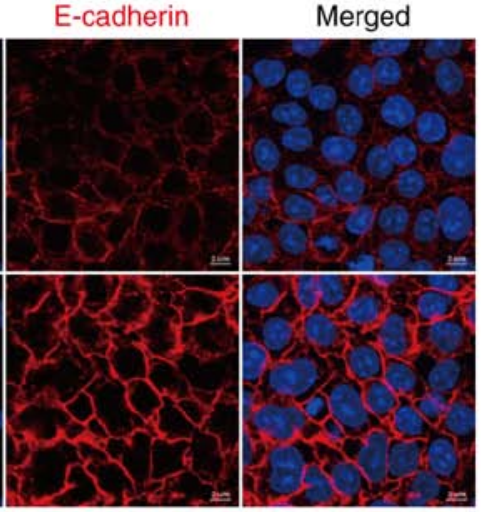

b

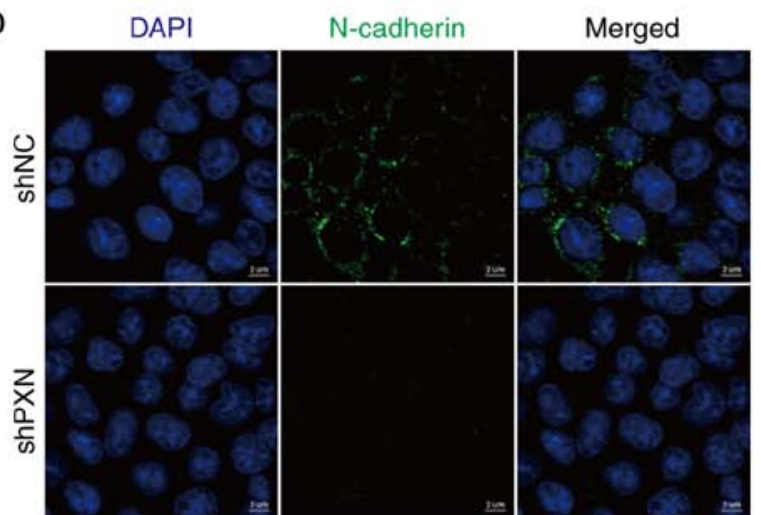

B

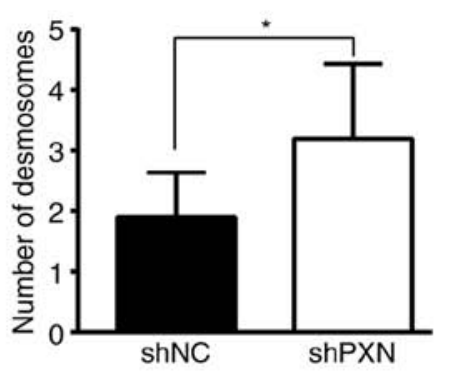

C

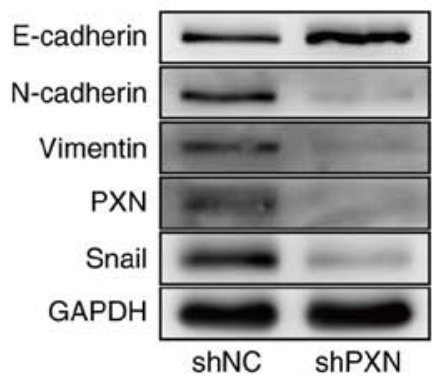
cell ultrastructure was observed by TEM. The number of microvilli was sharply decreased in (b) the shPXN group compared with (a) the shNC group (original magnification, $x 10,000$ ). The enlarged figure in (a) shows a glandular structure (original magnification, x20,000). (c and d) The junctions between tumours (red triangle, desmosome; yellow triangle, tight junction; original magnification, x60,000). (B) Desmosomes in ten random images were counted. The data are presented as the mean \pm SD. (C) The knockdown of PXN increased E-cadherin expression and decreased N-cadherin, vimentin and Snail expression. The expression of the aforementioned markers was detected by western blot analysis; the histogram represents the relative protein expression. (D) The immunofluorescence staining revealed that knockdown of PXN upregulated the expression of (a, red) E-cadherin and downregulated the expression of (b, green) $\mathrm{N}$-cadherin and (c, green) vimentin. Nuclear staining with DAPI (blue). P-values were obtained by Student's t-test. ${ }^{*} \mathrm{P}<0.05,{ }^{* *} \mathrm{P}<0.01$. PXN, paxillin; EMT, epithelial-mesenchymal transition; DAPI, 4',6-diamidino-2-phenylindole.

Knockdown of PXN inhibits EMT through the ERK signalling pathway. To verify the hypothesis that PXN regulates EMT through the ERK signalling pathway, two drugs were applied to activate (hEGF) or inhibit (EI; SCH-772984) the ERK signalling pathway and validated their effects in cell experiments.
The viabilities of shNC and shPXN cells under drug treatments in the serum-free state at 0,24 and $48 \mathrm{~h}$ were assessed. There was no significant difference in proliferation and viability of either cells under different treatments $(\mathrm{F}=0.732$, $\mathrm{P}=0.601$, Fig. S2), which indicated that within 48 h, tumour 

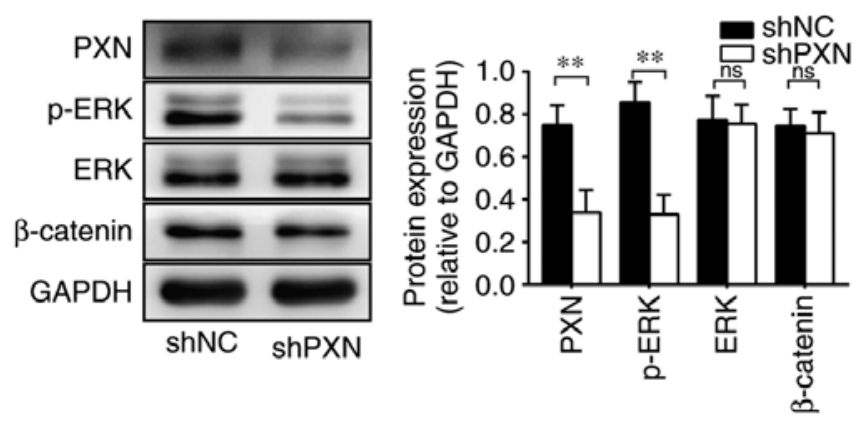

Figure 5. PXN regulates the ERK signalling pathway. The downregulation of PXN induced the expression of p-ERK, decreasing ERK activation, while the total ERK and $\beta$-catenin levels remained stable. The expression levels of p-ERK, ERK and $\beta$-catenin were assessed by western blot analysis. The histogram represents the relative protein expression. ${ }^{* *} \mathrm{P}<0.01$. PXN, paxillin; ERK, extracellular regulated protein kinase; p-, phosphorylated; ns, no significance.

proliferation had little effect on the results of migration and invasion assays. Then, we measured changes in the migration and invasion abilities of SW480 cells. First, similar to the aforementioned results (Fig. 2F-H), the downregulation of PXN effectively attenuated tumour migration and invasion. Second, activation of the ERK signalling pathway by hEGF increased the migration and invasion abilities of both groups. In contrast, the migration and invasion abilities of both groups were decreased following treatment with EI. Finally, although migration and invasion abilities were reduced by PXN knockdown, they were restored by ERK activation and further attenuated by combination treatment with EI. Consistent results were obtained in wound healing (Fig. 6A-a), migration (Fig. 6A-b) and invasion (Fig. 6A-c) assays, despite differences in the proportions of each group of cells in the three experiments (Fig. 6B a-c).

Then, the expression levels of ERK and EMT markers were investigated. Previous experimental results confirmed that the downregulation of PXN decreased p-ERK levels and the EMT process. These experiments were repeated to make the entire study more rigorous and confirm the previous conclusions. As revealed in Fig. 6C, in the shNC group, the expression levels of $\mathrm{N}$-cadherin, vimentin and Snail decreased with the decrease in p-ERK due to the application of EI; however, E-cadherin expression exhibited an increasing trend. In the shPXN group, the expression levels of mesenchymal markers that had already been reduced due to PXN knockdown were increased by the effect of the ERK activator, accompanied by a decreased expression of E-cadherin, and EI further suppressed the expression of these three mesenchymal markers. Notably, changes in the expression of these three markers were consistent with changes in only p-ERK expression, as the total ERK levels remained unchanged.

Collectively, these data indicated that the knockdown of PXN inhibited EMT through downregulation of the ERK signalling pathway.

\section{Discussion}

Approximately $20 \%$ of patients with CRC present with distant metastasis at the time of diagnosis, and approximately $40 \%$ of patients with localized disease develop metastases within 5 years after surgery $(1,16)$. Metastasis is a multi-step process, and its first step requires the local invasion of tumour cells (3). PXN, a multi-domain focal adhesion adaptor protein, contributes to recruiting structural and signalling molecules involved in cell movement and invasion (11).

By analysing clinical data, it was confirmed that PXN expression was positively associated to the clinicopathological parameters of metastasis. Moreover, patients who developed distant site recurrence after radical surgery were more likely to express high levels of PXN. A previous study reported that PXN expression in lymph node metastases was more intense than that in primary adenocarcinoma (17). Similarly, Ikuta et al (18) confirmed the significance of CAF expression in metastatic lymph nodes for a poor prognosis, while the level of genes related to the PXN signalling pathways was higher in CAFs than in normal fibroblasts (19), which may be a potential mechanism by which PXN promotes metastasis. In the present study, it was further revealed that PXN expression was higher in liver metastasis compared with the paired primary tumour. However, in a recent study (20), the expression of phosphorylated PXN was decreased in liver metastasis compared with the matched paired primary cancer and correlated with poor histological grade in CRC patients. This finding may be because the phosphorylation status of several protein kinases is different in primary and metastatic matched lesions, due to the activation of various signalling pathways (21). All of the aforementioned results indicated that PXN plays an important and complex role in CRC metastasis.

The in vitro and in vivo experiments in the present study demonstrated that the knockdown of PXN inhibited cell migration and invasion. PXN, a downstream binding partner of FAK/Src, performs critical functions in coordinating the ligation of integrin and the extracellular matrix (ECM) (22). The phosphorylation of PXN alters the organization of focal adhesions and increases cell motility (23). The significant effect of PXN on metastasis was directly reflected and evidenced in the ultrastructural changes observed with PXN knockdown. The appearance of numerous microvilli on the surface of tumour cells is characteristic of active cells in general (24). More microvilli form in highly metastatic colon carcinoma cells than in weakly metastatic ones, indicating that the presence of an increased number of microvilli closely correlates with the metastatic ability of CRC cells (25). PXN regulates cell polarity and the cytoskeleton through binding to the regulators and effectors of Rho GTPases (26). By inhibiting Rho signalling, ERK1/2 signalling pathway activation leads to malignant transformations, such as the transition of ruffles into active microvilli (27). The present results indicated that the knockdown of PXN decreased the number of microvilli and deactivated Erk1/2, accompanied by a decrease in migration and invasion abilities. In addition, the downregulation of PXN increased the number of desmosomes and led to clearer junction structures. Desmosomes, a type of intercellular junction, enhance adhesion between epithelial cells, and a decreased number of desmosomes indicates the promotion of cell invasion (28). When the normal epithelium is transformed to a malignant epithelium, desmosomes become unstable and easily disrupted, facilitating cell separation (29). We did not further explore how PXN affects desmosomes; however, the 
A

a
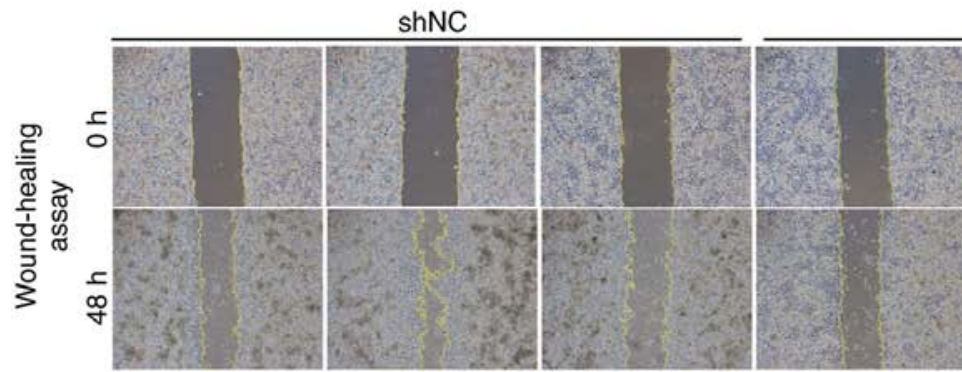

ShPXN
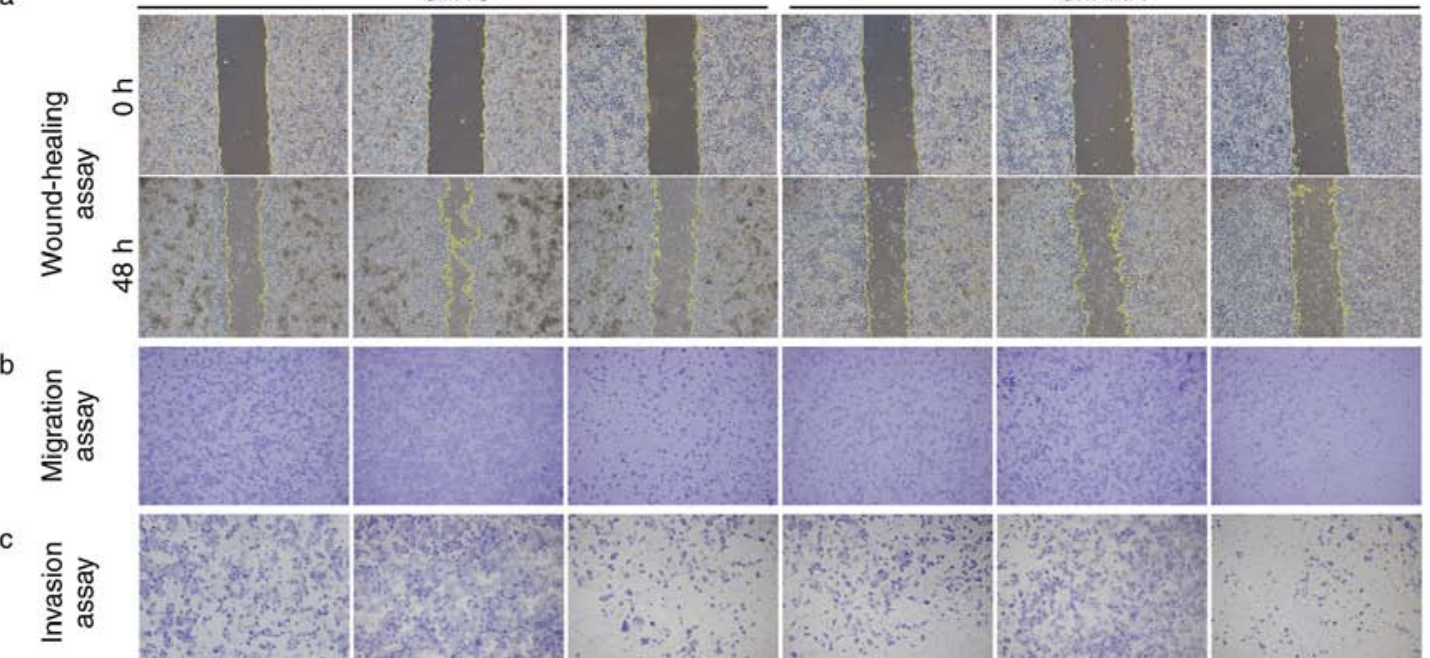

B a
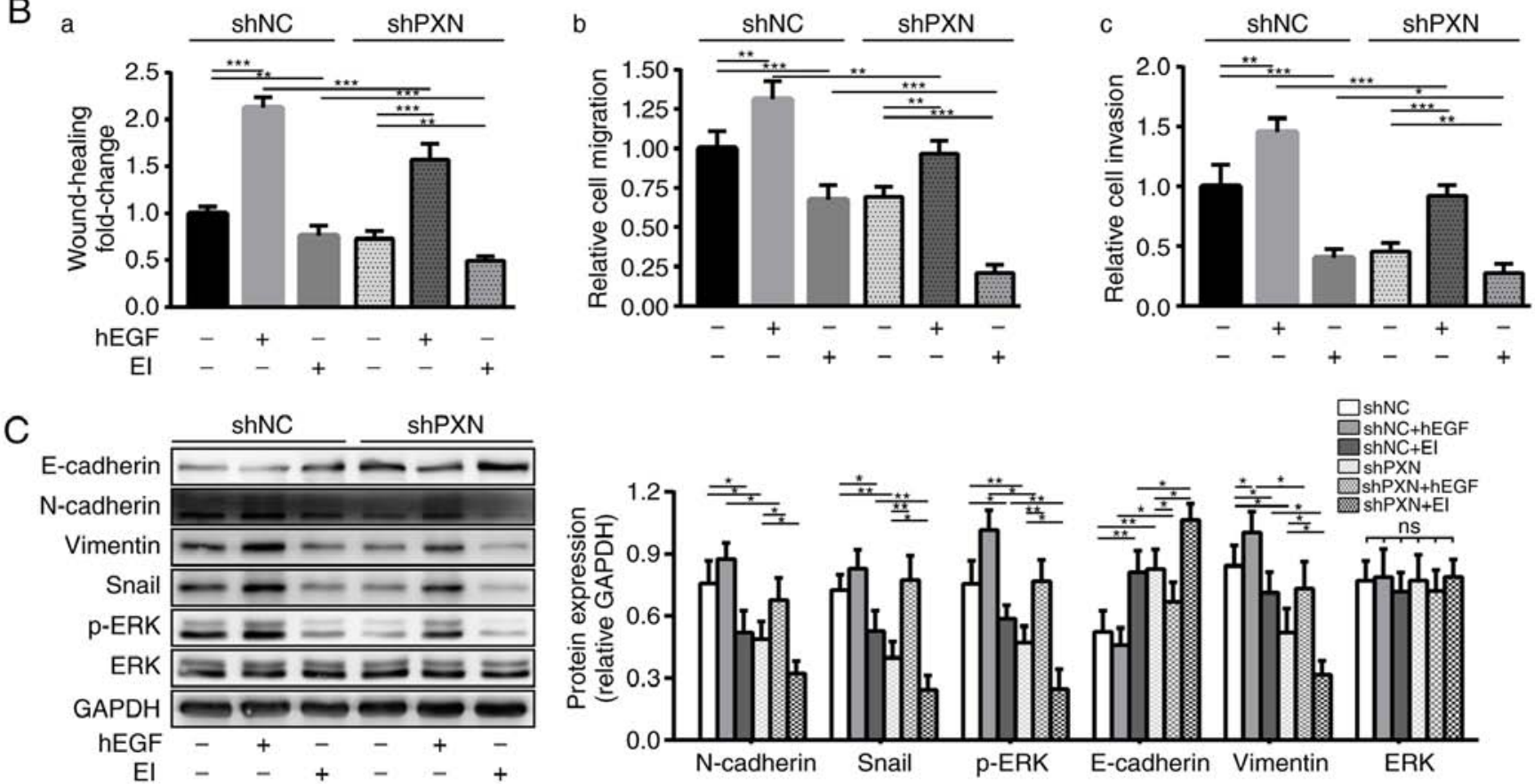

Figure 6. Knockdown of PXN suppresses the migration and invasion abilities of SW480 cells by inhibiting EMT through the ERK signalling pathway. SW480 cells transfected with shNC and shPXN were treated with an ERK activator (hEGF, $0.6 \mu \mathrm{g} / \mathrm{ml}$ ) or inhibitor (SCH-772984; EI, 0.5 $\mu \mathrm{g} / \mathrm{ml}$ ). (A) The migration and invasion abilities of cells were detected using (a) wound healing, Transwell (b) migration and (c) invasion assays and are respectively illustrated by (B, a-c) histograms. The histograms represent the wound width change and the number of migrated and invaded cells relative to the shNC group. (C) Activation of the ERK signalling pathways promoted PXN-mediated EMT. In contrast, inhibition of the ERK signalling pathways further suppressed the EMT process. The expression levels of p-ERK, ERK and EMT markers were analysed by western blot assay. The data are presented as the mean \pm SD of at least three independent experiments. The histograms of the WB experiments represent the relative protein expression. P-values were obtained using Student's t-test. ${ }^{*} \mathrm{P}<0.05,{ }^{* *} \mathrm{P}<0.01,{ }^{* * *} \mathrm{P}<0.001$. PXN, paxillin; EMT, epithelial-mesenchymal transition; ERK, extracellular regulated protein kinase; p-, phosphorylated; EI, ERK inhibitor; ns, no significance.

change in the number of desmosomes is important evidence that PXN promotes cell dissemination.

Previous studies have reported that PXN plays different roles in EMT in diverse tissues and tumours. PXN $\delta$, which lacks the key phosphorylation sites of PXN at Y31 and Y118, was revealed to downregulate TGF- $\beta$-induced EMT in normal murine mammary epithelial cells (30). Upregulation of the ERK-FAK-PXN axis led to the induction of type 2 EMT-like changes during corneal epithelial wound healing (31).
In trabecular meshwork cells, the knockdown of PXN blocked EMT-like alterations in cellular characteristics (32). Although the depletion of PXN in breast cancer cells led to an elongated morphology, it decreased the ability of cells to undergo trans-endothelial migration (33). In the present study, inhibiting the expression of PXN increased the expression of E-Cadherin and enhanced the adherens junction, suggesting that the knockdown of PXN suppressed EMT progression. In addition, as an adaptor protein in the integrin signalling 
pathway, PXN has been revealed to provide a necessary link in the FAK-dependent survival pathway and contribute to anoikis resistance (34), which promotes EMT and tumour metastasis (35).

In the present study, the Wnt and ERK signalling pathways were identified as responsible for the underlying mechanisms by which PXN regulated EMT. The expression of $\beta$-catenin, a typical marker of the Wnt pathway that regulates Snail (36), was not altered with PXN knockdown; however, p-ERK expression displayed a decreasing trend. Other proteins that regulate EMT processes via the ERK signalling pathway in CRC have been previously reported $(8,37)$. In addition, several studies have demonstrated that PXN mutation led to a reduction in cell motility and survival mediated by ERK1/2 (38), and PXN regulated cell proliferation by modulating ERK signalling and its subsequent downstream effects (39). ERK has been revealed to be associated with PXN phosphorylation and mediate epithelial morphogenesis, indicating the important role of the ERK-PXN association in cell adhesion and migration. However, the authors did not verify EMT (40). Based on these findings, it was hypothesized that the ERK signalling pathway plays an important role in the mechanism by which PXN regulates EMT. Following PXN downregulation, ERK was inactivated to a degree consistent with the decreased expression of EMT markers. This finding indicated that reactivation of the ERK pathway restored EMT and that PXN knockdown combined with an ERK inhibitor further blocked the ERK pathway. Changes to cellular migration and invasion were also in accordance with the activation status of ERK. However, changes in the expression of EMT markers were not completely consistent with changes in cell migration and invasion, which may be attributed to the fact that PXN is one of the various regulators of the ERK signalling pathway $(23,40)$. Furthermore, there were several limitations in present study, such as the effect of PXN on the specific transcription mechanism of EMT and the tumour microenvironment during CRC metastasis, which require further exploration in a following study.

In addition, published studies have indicated that PXN is not only associated with the microtubule network and the polarization and motility of lymphoid cells $(41,42)$, but is also involved in regulating the migration of antigen-presenting cells, such as macrophages, neutrophils and dendritic cells (43-45). The knockdown of PXN suppressed the invasion of colon cancer cell by inhibiting M2 macrophage polarization (46), which indicates that PXN could regulate tumour metastasis through immune cells.

In summary, the present findings indicated that the knockdown of PXN inhibited EMT through the ERK signalling pathway, thereby reducing the ability of CRC to invade and metastasize. PXN knockdown may represent a future therapeutic strategy to prevent the EMT-associated progression and invasion of CRC.

\section{Acknowledgements}

The authors would like to thank Professor Ding-Fang Bu for technical assistance with the WB assay, Mrs. Xu Zhang for technical guidance with TEM, and Mrs. Yuanyuan Ma for animal study assistance.

\section{Funding}

No funding was received.

\section{Availability of data and materials}

All data generated or analysed during this study are included in this article.

\section{Authors' contributions}

JZha and XW designed this study. LW and JZhu completed the experiments. LW and XZ wrote the manuscript. JW, JH and TY performed sample collection. XZ, YM, TW and SC analysed the data. All authors read and approved the final version of the manuscript.

\section{Ethics approval and consent to participate}

The study protocol was approved by Peking University First Hospital Biomedical Research Ethics Committee (no. 2018-15). All patients related to this study signed an informed consent agreement before surgery. The animal experiments were approved by the Laboratory Animal Ethics Committee of Peking University First Hospital (no. 2015-55).

\section{Patient consent for publication}

Not applicable.

\section{Competing interests}

The authors declare that they have no competing interests.

\section{References}

1. Torre LA, Bray F, Siegel RL, Ferlay J, Lortet-Tieulent J and Jemal A: Global cancer statistics, 2012. CA Cancer J Clin 65: 87-108, 2015.

2. Siegel RL, Miller KD, Fedewa SA, Ahnen DJ, Meester R, Barzi A and Jemal A: Colorectal cancer statistics, 2017. CA Cancer J Clin 67: 177-193, 2017.

3. Cao H, Xu E, Liu H, Wan L and Lai M: Epithelial-mesenchymal transition in colorectal cancer metastasis: A system review. Pathol Res Pract 211: 557-569, 2015.

4. Mizukoshi K, Okazawa Y, Haeno H, Koyama Y, Sulidan K, Komiyama H, Saeki H, Ohtsuji N, Ito Y, Kojima Y, et al: Metastatic seeding of human colon cancer cell clusters expressing the hybrid epithelial/mesenchymal state. Int J Cancer 146: 2547-2562, 2019.

5. Lampropoulos P, Zizi-Sermpetzoglou A, Rizos S, Kostakis A, Nikiteas $\mathrm{N}$ and Papavassiliou AG: TGF-beta signalling in colon carcinogenesis. Cancer Lett 314: 1-7, 2012.

6. Li T, Zhu J, Wang X, Chen G, Sun L, Zuo S, Zhang J, Chen S, Ma J, Yao Z, et al: Long non-coding RNA lncTCF7 activates the Wnt/ $\beta$-catenin pathway to promote metastasis and invasion in colorectal cancer. Oncol Lett 14: 7384-7390, 2017.

7. Hong SK, Park JR, Kwon OS, Kim KT, Bae GY and Cha HJ: Induction of integrin $\beta 3$ by sustained ERK activity promotes the invasiveness of TGF $\beta$-induced mesenchymal tumor cells. Cancer Lett 376: 339-346, 2016.

8. Tanahashi T, Osada S, Yamada A, Kato J, Yawata K, Mori R, Imai H, Sasaki Y, Saito S, Tanaka Y, et al: Extracellular signal-regulated kinase and Akt activation play a critical role in the process of hepatocyte growth factor-induced epithelial-mesenchymal transition. Int J Oncol 42: 556-564, 2013. 
9. Smith BN, Burton LJ, Henderson V, Randle DD, Morton DJ, Smith BA, Taliaferro-Smith L, Nagappan P, Yates C, Zayzafoon $\mathrm{M}$, et al: Snail promotes epithelial mesenchymal transition in breast cancer cells in part via activation of nuclear ERK2. PLoS One 9: e104987, 2014.

10. Deakin NO and Turner CE: Paxillin comes of age. J Cell Sci 121: 2435-2444, 2008.

11. López-Colomé, Lee-Rivera I, Benavides-Hidalgo R and López E: Paxillin: A crossroad in pathological cell migration. J Hematol Oncol 10: 50, 2017.

12. Zhao CJ, Du SK, Dang XB and Gong M: Expression of paxillin is correlated with clinical prognosis in colorectal cancer patients. Med Sci Monit 21: 1989-1995, 2015.

13. Du C, Wang X, Zhang J, Liu X, Zhu J and Liu Y: Paxillin is positively correlated with the clinicopathological factors of colorectal cancer, and knockdown of Paxillin improves sensitivity to cetuximab in colorectal cancer cells. Oncol Rep 35: 409-417, 2016.

14. Chen DL, Wang DS, Wu WJ, Zeng ZL, Luo HY, Qiu MZ, Ren C, Zhang DS, Wang ZQ, Wang FH, et al: Overexpression of paxillin induced by miR-137 suppression promotes tumor progression and metastasis in colorectal cancer. Carcinogenesis 34: 803-811, 2013.

15. Joo D, Woo JS, Cho KH, Han SH, Min TS, Yang DC and Yun $\mathrm{CH}$ : Biphasic activation of extracellular signal-regulated kinase (ERK) $1 / 2$ in epidermal growth factor (EGF)-stimulated SW480 colorectal cancer cells. BMB Rep 49: 220-225, 2016.

16. Takahashi E: The regulation of epithelial mesenchymal transition in ocular disorders. Nippon Ganka Gakkai Zasshi 120: 783-790, 2016 (In Japanese).

17. Yang HJ, Chen JZ, Zhang WL and Ding YQ: Focal adhesion plaque associated cytoskeletons are involved in the invasion and metastasis of human colorectal carcinoma. Cancer Invest 28: 127-134, 2010

18. Ikuta D, Miyake T, Shimizu T, Sonoda H, Mukaisho KI, Tokuda A, Ueki T, Sugihara $\mathrm{H}$ and Tani M: Fibrosis in metastatic lymph nodes is clinically correlated to poor prognosis in colorectal cancer. Oncotarget 9: 29574-29586, 2018.

19. Takahashi H, Sakakura K, Kawabata-Iwakawa R, Rokudai S, Toyoda M, Nishiyama M and Chikamatsu K: Immunosuppressive activity of cancer-associated fibroblasts in head and neck squamous cell carcinoma. Cancer Immunol Immunother 64 1407-1417, 2015.

20. Mantonakis E, Giaginis C, Pikoulis E, Felekouras E, Patsouris E, Liakakos T, Papalampros E and Theocharis S: FAK, Src and p-Paxillin expression is decreased in liver metastasis of colorectal carcinoma patients. J BUON 22: 1097-1106, 2017.

21. Belluco C, Mammano E, Petricoin E, Prevedello L, Calvert V, Liotta L, Nitti D and Lise M: Kinase substrate protein microarray analysis of human colon cancer and hepatic metastasis Clin Chim Acta 357: 180-183, 2005

22. Deakin NO, Pignatelli J and Turner CE: Diverse roles for the paxillin family of proteins in cancer. Genes Cancer 3: 362-370, 2012.

23. Devreotes P and Horwitz AR: Signaling networks that regulate cell migration. Cold Spring Harb Perspect Biol 7: a005959, 2015

24. Meng Y, Lu Z, Yu S, Zhang Q, Ma Y and Chen J: Ezrin promotes invasion and metastasis of pancreatic cancer cells. J Transl Med 8: 61, 2010

25. Ren J, Hamada J, Okada F, Takeichi N, Morikawa K, Hosokawa M and Kobayashi H: Correlation between the presence of microvilli and the growth or metastatic potential of tumor cells. Jpn J Cancer Res 81: 920-926, 1990.

26. Hodge RG and Ridley AJ: Regulating Rho GTPases and their regulators. Nat Rev Mol Cell Biol 17: 496-510, 2016.

27. Barros JC and Marshall CJ: Activation of either ERK1/2 or ERK 5 MAP kinase pathways can lead to disruption of the actin cytoskeleton. J Cell Sci 118: 1663-1671, 2005.

28. Alroy J, Pauli BU and Weinstein RS: Correlation between numbers of desmosomes and the aggressiveness of transitional cell carcinoma in human urinary bladder. Cancer 47: 104-112, 1981.
29. Collins JE, Taylor I and Garrod DR: A study of desmosomes in colorectal carcinoma. Br J Cancer 62: 796-805, 1990.

30. Tumbarello DA, Brown MC, Hetey SE and Turner CE: Regulation of paxillin family members during epithelial-mesenchymal transformation: A putative role for paxillin delta. J Cell Sci 118: 4849-4863, 2005

31. Kowtharapu BS, Murin R, Jünemann AGM and Stachs O: Role of corneal stromal cells on epithelial cell function during wound healing. Int J Mol Sci 19: 464, 2018

32. Takahashi E, Inoue T, Fujimoto T, Kojima $S$ and Tanihara H: Epithelial mesenchymal transition-like phenomenon in trabecular meshwork cells. Exp Eye Res 118: 72-79, 2014.

33. Deakin NO and Turner CE: Distinct roles for paxillin and Hic-5 in regulating breast cancer cell morphology, invasion, and metastasis. Mol Biol Cell 22: 327-341, 2011.

34. Zouq NK, Keeble JA, Lindsay J, Valentijn AJ, Zhang L, Mills D, Turner CE, Streuli CH and Gilmore AP: FAK engages multiple pathways to maintain survival of fibroblasts and epithelia: Differential roles for paxillin and p130Cas. J Cell Sci 122: 357-367, 2009.

35. Simpson CD, Anyiwe K and Schimmer AD: Anoikis resistance and tumor metastasis. Cancer Lett 272: 177-185, 2008.

36. Stemmer V, de Craene B, Berx G and Behrens J: Snail promotes Wnt target gene expression and interacts with beta-catenin. Oncogene 27: 5075-5080, 2008.

37. Ha GH, Park JS and Breuer EK: TACC3 promotes epithelial-mesenchymal transition (EMT) through the activation of PI3K/Akt and ERK signaling pathways. Cancer Lett 332: 63-73, 2013.

38. Subauste MC, Pertz O, Adamson ED, Turner CE, Junger S and Hahn KM: Vinculin modulation of paxillin-FAK interactions regulates ERK to control survival and motility. J Cell Biol 165: 371-381, 2004

39. Sen A, De Castro I, Defranco DB, Deng FM, Melamed J, Kapur P, Raj GV, Rossi R and Hammes SR: Paxillin mediates extranuclear and intranuclear signaling in prostate cancer proliferation. J Clin Invest 122: 2469-2481, 2012.

40. Ishibe S, Joly D, Zhu X and Cantley LG: Phosphorylationdependent paxillin-ERK association mediates hepatocyte growth factor-stimulated epithelial morphogenesis. Mol Cell 12: $1275-1285,2003$.

41. Herreros L, Rodríguez-Fernandez JL, Brown MC, Alonso-Lebrero JL, Cabañas C, Sánchez-Madrid F, Longo N, Turner CE and Sanchez-Mateos P: Paxillin localizes to the lymphocyte microtubule organizing center and associates with the microtubule cytoskeleton. J Biol Chem 275: 26436-26440, 2000.

42. Romanova LY, Hashimoto S, Chay KO, Blagosklonny MV, Sabe $\mathrm{H}$ and Mushinski JF: Phosphorylation of paxillin tyrosines 31 and 118 controls polarization and motility of lymphoid cells and is PMA-sensitive. J Cell Sci 117: 3759-3768, 2004.

43. Rose DM, Han J and Ginsberg MH: Alpha4 integrins and the immune response. Immunol Rev 186: 118-124, 2002.

44. Rhee I, Zhong MC, Reizis B, Cheong C and Veillette A: Control of dendritic cell migration, T cell-dependent immunity, and autoimmunity by protein tyrosine phosphatase PTPN12 expressed in dendritic cells. Mol Cell Biol 34: 888-899, 2014.

45. Parsons SA, Sharma R, Roccamatisi DL, Zhang H, Petri B, Kubes P, Colarusso P and Patel KD: Endothelial paxillin and focal adhesion kinase (FAK) play a critical role in neutrophil transmigration. Eur J Immunol 42: 436-446, 2012.

46. Zhang LL, Zhang LF and Shi YB: Down-regulated paxillin suppresses cell proliferation and invasion by inhibiting M2 macrophage polarization in colon cancer. Biol Chem 399: $1285-1295,2018$

This work is licensed under a Creative Commons Attribution-NonCommercial-NoDerivatives 4.0 International (CC BY-NC-ND 4.0) License. 